\title{
Reactivity of Monolayer-Protected Gold Nanoclusters at Dye-Sensitized Liquid/Liquid Interfaces
}

\author{
Bin Su, Nicolas Eugster, and Hubert H. Girault* \\ Contribution from the Laboratoire d'Electrochimie Physique et Analytique, \\ Ecole Polytechnique Fédérale de Lausanne, CH-1015 Lausanne, Switzerland
}

Received April 14, 2005; E-mail: hubert.girault@epfl.ch

\begin{abstract}
Hexanethiolate monolayer-protected gold nanoclusters (MPCs) were used as redox quenchers at the polarizable water/1,2-dichloroethane (DCE) interface. Photocurrent responses originating from the heterogeneous quenching of photoexcited water soluble porphyrin complexes by MPCs dissolved in the DCE phase were observed. As MPCs can function as both electron acceptors and donors, the photocurrent results from the superposition of two simultaneous processes, which correspond to the oxidation and reduction of MPCs. The magnitude of the net photocurrent is essentially determined by the balance of the kinetics of these two processes, which can be controlled by tuning the Galvani potential difference between the two phases. We show that, within the available potential window, the apparent electron-transfer rate constants follow classical Butler-Volmer dependence on the applied potential difference.
\end{abstract}

\section{Introduction}

An important property of metallic nanoparticles is their propensity to act as electron donors or acceptors. At ambient temperatures, successive single electron-transfer phenomena have been observed by voltammetry for alkanethiolate monolayer-protected gold nanoclusters (MPCs, prepared by Brust reaction ${ }^{1,2}$ ) either dissolved in electrolyte solutions or attached to electrodes as films. ${ }^{3-6} \mathrm{~A}$ series of evenly spaced voltammetric current peaks are observed, which are characteristic of diffusion controlled redox processes. This electrochemical behavior, sometimes designated "quantized double layer" (QDL) charging, arises from the extremely small (sub-attoFarad) double layer capacitances of the coated nanoparticles. ${ }^{7}$ The QDL charging happens with metallic nanoparticles as they encompass the transition between bulk and molecular regimes.

It has been reported that MPCs can be electrolytically charged to the desired valence states at controllable potentials. ${ }^{8}$ The charged MPCs are stable in solution for hours, can be isolated as solid form, and retain most of their charges upon redispersion. The electronic charge stored on the MPCs endows them with a redox activity similar to that of a multivalent redox species.

(1) Brust, M.; Walker, M.; Bethell, D.; Schiffrin, D. J.; Whyman, R. J. Chem. Soc. Chem. Commun. 1994, 801-802.

(2) Brust, M.; Fink, J.; Bethell, D.; Schiffrin, D. J.; Kiely, C. J. Chem. Soc., Chem. Commun. 1995, 1655-1656.

(3) Ingram, R. S.; Hostetler, M. J.; Murray, R. W.; Schaaff, T. G.; Khoury, J.; Whetten, R. L.; Bigioni, T. P.; Guthrie, D. K.; First, P. N. J. Am. Chem. Soc. 1997, 119, 9279-9280.

(4) Chen, S.; Ingrma, R. S.; Hostetler, M. J.; Pietron, J. J.; Murray, R. W.; Schaaff, T. G.; Khoury, J. T.; Alvarez, M. M.; Whetten, R. L. Science 1998, 280, 2098-2101.

(5) Chen, S. J. Electroanal. Chem. 2004, 574, 153-165.

(6) Templeton, A. C.; Wuelfing, W. P.; Murray, R. W. Acc. Chem. Res. 2000 , 33, 27-36.

(7) Chen, S.; Murray, R. W.; Feldberg, S. W. J. Phys. Chem. B 1998, 102, 9898-9907.

(8) Pietron, J. J.; Hicks, J. F.; Murray, R. W. J. Am. Chem. Soc. 1999, 121, $5565-5570$
The reactivity of MPCs toward electron transfer has been investigated in solution, ${ }^{4,8-12}$ in assemblies and films ${ }^{13-27}$ and at the air/water ${ }^{28}$ and liquid/liquid ${ }^{11,29}$ interfaces. Photochemical experiments have also demonstrated several examples of excitedstate quenching of surface-bound dyes by the metallic MPC cores through energy transfer ${ }^{30-32}$ or electron transfer. ${ }^{33}$

(9) Hicks, J. F.; Templeton, A. C.; Chen, S.; Sheran, K. M.; Jasti, R.; Murray R. W.; Debord, J.; Schaaff, T. G.; Whetten, R. L. Anal. Chem. 1999, 71 $3703-3711$.

(10) Hicks, J. F.; Miles, D. T.; Murray, R. W. J. Am. Chem. Soc. 2002, 124, $13322-13328$.

(11) Quinn, B. M.; Liljeroth, P.; Kontturi, K. J. Am. Chem. Soc. 2002, 124, 12915-12921.

(12) Quinn, B. M.; Liljeroth, P.; Ruiz, V.; Laaksonen, T.; Kontturi, K. J. Am. Chem. Soc. 2003, 125, 6644-6645.

(13) Chen, S. J. Am. Chem. Soc. 2000, 122, 7420-7421.

(14) Chen, S.; Murray, R. W. J. Phys. Chem. B 1999, 103, 9996-10000.

(15) Brennan, J. L.; Branham, M. R.; Hicks, J. F.; Osisek, A. J.; Donkers, R. L.; Georganopoulou, D. G.; Murray, R. W. Anal. Chem. 2004, 76, 56115619.

(16) Chen, S. Langmuir 2001, 17, 2878-2884

(17) Chen, S. Langmuir 2001, 17, 6664-6668

(18) Chen, S.; Pei, R. J. Am. Chem. Soc. 2001, 123, 10607-10615.

(19) Hicks, J. F.; Zamborini, F. P.; Osisek, A. J.; Murray, R. W. J. Am. Chem. Soc. 2001, 123, 7048-7053.

(20) Hicks, J. F.; Zamborini, F. P.; Murray, R. W. J. Phys. Chem. B 2002, 106, $7751-7757$.

(21) Wuelfing, W. P.; Murray, R. W. J. Phys. Chem. B 2002, 106, 3139-3145.

(22) Wuelfing, W. P.; Green, S. J.; Pietron, J. J.; Cliffel, D. E.; Murray, R. W. J. Am. Chem. Soc. 2000, 122, 11465-11472.

(23) Liljeroth, P.; Quinn, B. M.; Ruiz, V.; Kontturi, K. Chem. Commun. 2003, $1570-1571$.

(24) Liljeroth, P.; Vanmaekelbergh, D.; Ruiz, V.; Kontturi, K.; Jiang, H.; Kauppinen, E.; Quinn, B. M. J. Am. Chem. Soc. 2004, 126, 7126-7132.

(25) Zamborini, F. P.; Hicks, J. F.; Murray, R. W. J. Am. Chem. Soc. 2000, $122,4514-4515$

(26) Zamborini, F. P.; Leopold, M. C.; Hicks, J. F.; Kulesza, P. J.; Malik, M A.; Murray, R. W. J. Am. Chem. Soc. 2002, 124, 8958-8964.

(27) Lee, W.-Y.; Hostetler, M. J.; Murray, R. W.; Majda, M. Isr. J. Chem. 1997, 37, 213-223.

(28) Yang, Y.; Pradhan, S.; Chen, S. J. Am. Chem. Soc. 2004, 126, 76-77.

(29) Georganopoulou, D. G.; Mirkin, M. V.; Murray, R. W. Nano Lett. 2004 4, $1763-1767$.

(30) Templeton, A. C.; Cliffel, D. E.; Murray, R. W. J. Am. Chem. Soc. 1999 $121,7081-7089$

(31) Aguila, A.; Murray, R. W. Langmuir 2000, 16, 5949-5954

(32) Huang, T.; Murray, R. W. Langmuir 2002, 18, 7077-7081. 
In this work, we use hexanethiolate monolayer-protected nanoclusters of gold as redox quenchers for heterogeneous photocurrent generation at the polarized water/1,2-dichloroethane (DCE) interface. Photocurrent responses originating from the heterogeneous quenching of photoexcited water soluble porphyrin complexes by MPCs present in the organic phase were investigated. As the MPCs can function as both electron acceptors and electron donors, the reduction and oxidation of MPCs by photoexcited porphyrin complexes can occur simultaneously. The photocurrent results from the superposition of two opposite processes whose proportion can be tuned by the Galvani potential difference across the interface. The magnitude of the net photocurrent is determined by the balance of the kinetics of these two reactions. Within the available potential window, the apparent electron-transfer rate constants obey classical Butler-Volmer dependence on the applied potential difference between the two phases. The rate of the electron transfer reaction was found to be faster than those previously reported in the case of conventional redox molecules at liquid/ liquid interfaces.

\section{Experimental Section}

Chemicals. All chemicals employed were of the highest commercially available purity and used as received. Zinc meso-tetrakis( $p$ sulfonatophenyl)porphyrin tetrasodium ( $\left.\mathrm{Na}_{4} \mathrm{ZnTPPS}\right)$ and zinc mesotetrakis $\left.(N \text {-methylpyridyl)porphyrin tetratosylate (ZnTMPyP(tosylate) })_{4}\right)$ were purchased from Porphyrin Products Inc. Hydrogen tetrachloroaurate trihydrate $\left(\mathrm{HAuCl}_{4} \cdot 3 \mathrm{H}_{2} \mathrm{O}\right)$, tetraoctylammonium bromide (TO$\mathrm{ABr})$, sodium tetrahydroborate $\left(\mathrm{NaBH}_{4}\right)$, and 1-hexanethiol $(\mathrm{C} 6-\mathrm{SH})$ were obtained from Aldrich. Ethanol, acetonitrile $\left(\mathrm{CH}_{3} \mathrm{CN}\right)$, dichloromethane, 1,2-dichloroethane (DCE), bis(triphenylphosphoranylidene)ammonium chloride (BTPPACl), lithium sulfate $\left(\mathrm{Li}_{2} \mathrm{SO}_{4}\right)$, and lithium chloride $(\mathrm{LiCl})$ were provided by Fluka. Lithium tetrakis(pentafluorophenyl)borate (LiTPFB) diethyl etherate was purchased from Boulder Scientific Company. The organic supporting electrolyte, bis(triphenylphosphoranylidene) ammonium tetrakis(pentafluorophenyl)borate (BTPPATPFB), was prepared as previously reported. ${ }^{34}$ The Au MPCs were prepared by the Brust reaction ${ }^{1,2}$ followed by a treatment yielding a population of MPCs with relatively uniform cores. ${ }^{10}$ The final product has a dominant population with an average core mass of $28 \mathrm{kDa}$, which corresponds to ca. $140 \mathrm{Au}$ atoms protected with ca. 53 hexanethiolate ligands. ${ }^{10}$ The population features a mean diameter of $1.6 \pm 0.4 \mathrm{~nm}$ in the metallic core, as determined by high-resolution transmission electron microscopy (Philips, CM 300).

Electrochemical Measurements. Electrochemical measurements at the liquid/liquid interface were carried out using a custom-built fourelectrode system, which was connected to a DS335 function generator (Stanford Research System) for cyclic voltammetry (CV) and a HiTek waveform generator (Hi-Tek Instruments PP-R1) for potentiostatic polarization. A three-compartment glass cell with a cross section of $1.53 \mathrm{~cm}^{2}$ was employed. The silver|silver sulfate $\left(\mathrm{Ag}_{2} \mathrm{SO}_{4}\right)$ and silver/ silver chloride $(\mathrm{Ag} / \mathrm{AgCl})$ reference electrodes were placed in two separate compartments featuring Luggin capillaries. The composition of the electrochemical cell is displayed in Figure 1. The Galvani potential difference $\left(\Delta_{\mathrm{o}}^{\mathrm{w}} \phi\right)$ was estimated by taking the formal ion transfer potential of the tetramethylammonium cation $\left(\mathrm{TMA}^{+}\right)$as 0.160 V.

Photocurrent Measurements. Photocurrent transients were measured under potentiostatic conditions. The illumination was carried out with the $442 \mathrm{~nm}$ line of a $\mathrm{He}-\mathrm{Cd}$ laser (Omnichrome S74) under

(33) Ipe, B. I.; Thomas, K. G.; Barazzouk, S.; Hotchandani, S.; Kamat, P. V. J. Phys. Chem. B 2002, 106, 18-21.

(34) Su, B.; Abid, J.-P.; Fermin, D. J.; Girault, H. H.; Hoffmannova, H.; Krtil, P.; Samec, Z. J. Am. Chem. Soc. 2004, 126, 915-919.

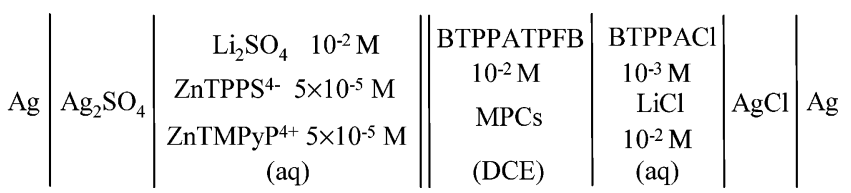

Figure 1. Cell employed for electrochemical and photocurrent measurements at water/DCE interfaces.
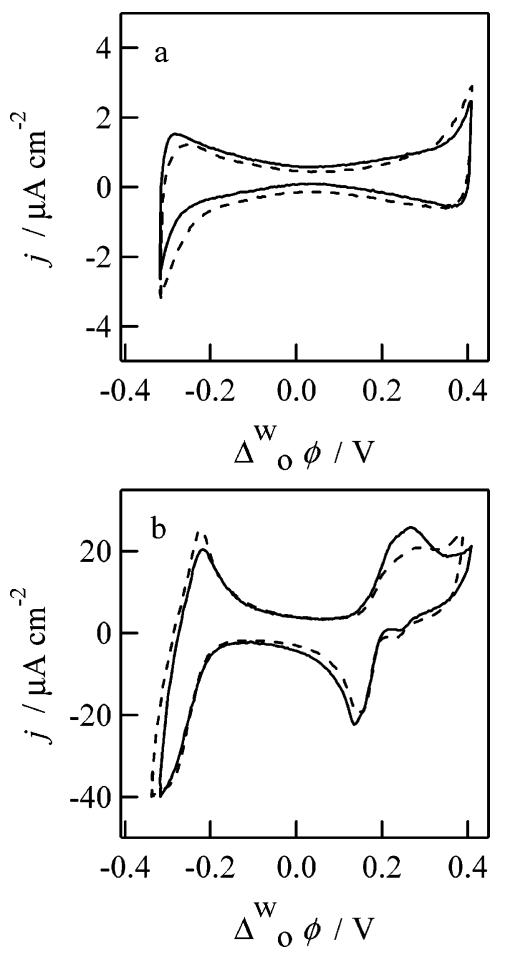

Figure 2. (a) $\mathrm{CVs}$ at the water/DCE interface in the absence (solid line) and presence (dashed line) of ca. $0.02 \mathrm{mM} \mathrm{Au} \mathrm{MPC} Z$ in DCE. (b) CVs of the porphyrin complex $\left(50 \mu \mathrm{M} \mathrm{ZnTPPS}^{4-}+50 \mu \mathrm{M} \mathrm{ZnTMPyP}^{4+}\right)$ at the water/DCE interface in the absence (solid line) and presence (dashed line) of ca. $0.02 \mathrm{mM} \mathrm{MPC}^{Z}$ in DCE. In all cases, the scan rate was $50 \mathrm{mV} \mathrm{s}^{-1}$.

transmission mode. The light was directed to the interface by passing through the upper aqueous phase in order to avoid light scattering by the MPCs in DCE. An optical shutter with an aperture time in the range of microseconds was used to control the illumination. The incident photon flux was quantitatively determined with a calibrated photodiode (model 70316, Thermo Oriel Instruments).

\section{Results and Discussion}

Photoinduced Electron Transfer between Porphyrin Complexes and MPCs. Figure 2a shows the polarizable potential windows of the water/DCE interface in the absence and presence of $\mathrm{MPC}^{Z}$ in DCE phase. The similarity of these two CV curves indicates that $\mathrm{MPC}^{Z}$ has no substantial effect on the electrochemical behavior of the water|DCE interface. The solid line in Figure $2 b$ is the $\mathrm{CV}$ response in the presence of the $\mathrm{ZnTPPS}^{4-}-\mathrm{ZnTMPyP}^{4+}$ complex in aqueous phase. The signals observed close to the ends of the potential window correspond to the transfer and adsorption of porphyrin ions and their counterions. ${ }^{35}$ Again, the addition of $\mathrm{MPC}^{Z}$ to the system did not result in substantial changes in the $\mathrm{CV}$ response. This indicates not only that the presence of $\mathrm{MPC}^{Z}$ has negligible effect on the specific adsorption of the porphyrin complex but also that no electron exchange occurs between $\mathrm{MPC}^{Z}$ and the

(35) Eugster, N.; Fermin, D. J.; Girault, H. H. J. Phys. Chem. B 2002, 106, $3428-3433$. 

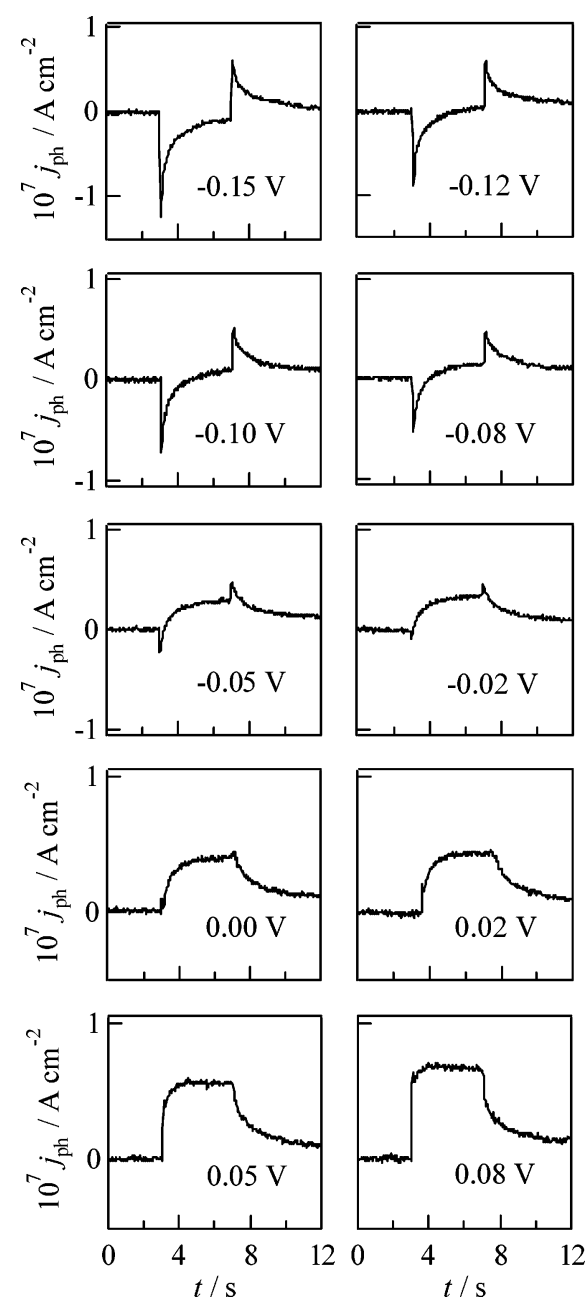

Figure 3. Photocurrent transient responses obtained in the presence of ca. $0.02 \mathrm{mM} \mathrm{MPC}{ }^{Z}$ in DCE and $50 \mu \mathrm{M} \mathrm{ZnTPPS}^{4-}-\mathrm{ZnTMPyP}^{4+}$ in water at various Galvani potential differences.

ground state of the porphyrin complex. As shown in Figure 2b, no faradaic responses are observed between -0.15 and $0.10 \mathrm{~V}$. The Galvani potential difference will be constrained within this potential region in all subsequent photocurrent measurements. In addition, it has been shown that the porphyrin complex exhibits nearly full interfacial coverage of the water/DCE boundary in this potential range. ${ }^{36}$

Photocurrent responses at various Galvani potential differences in the presence of the porphyrin complex in water and $\mathrm{MPC}^{Z}$ in DCE are displayed in Figure 3. As described in the literature, gold nanoparticles show limited photoactivity under UV-visible light, ${ }^{33}$ although photoinduced fusion and fragmentation of nanorods ${ }^{37-39}$ and near-IR luminescence of $\mathrm{MPCs}^{40-42}$ have been observed. The excited electrons in nanoparticles follow fast relaxations via electron-phonon coupling and phonon-phonon interaction of the lattice on the

(36) Fermin, D. J.; Duong, H. D.; Ding, Z.; Brevet, P.-F.; Girault, H. H. J. Am. Chem. Soc. 1999, 121, 10203-10210.

(37) Link, S.; Burda, C.; Mohamed, M. B.; Nikoobakht, B.; El-Sayed, M. A. J. Phys. Chem. A 1999, 103, 1165-1170.

(38) Link, S.; Burda, C.; Nikoobakht, B.; El-Sayed, M. A. J. Phys. Chem. B 2000, 104, 6152-6163.

(39) Link, S.; El-Sayed, M. A. J. Chem. Phys. 2001, 114, 2362-2368.

(40) Lee, D.; Donkers, R. L.; Wang, G.; Harper, A. S.; Murray, R. W. J. Am. Chem. Soc. 2004, 126, 6193-6199.

(41) Huang, T.; Murray, R. W. J. Phys. Chem. B 2001, 105, 12498-12502.

(42) Wilcoxon, J. P.; Martin, J. E.; Parsapour, F.; Wiedenman, B.; Kelley, D. F. J. Chem. Phys. 1998, 108, 9137-9143. picosecond time scale. ${ }^{43}$ On the other hand, ultrafast timeresolved spectroscopic measurements have demonstrated that the excited porphyrin complex undergoes an intermolecular quenching in less than 100 ps to reach a charge separated state, which can be involved in heterogeneous electron-transfer reactions at the water/DCE interface. ${ }^{44}$ Based on these considerations, we conclude that the photocurrent responses observed in Figure 3 arise from the heterogeneous electron transfer between the charge-separated state of the porphyrin complex and the ground-state MPC. As demonstrated in a recent paper, ${ }^{45}$ the DPV response of as-prepared MPCs in the DCE electrolyte solution features a series of well-resolved and evenly spaced current peaks about $230 \mathrm{mV}$ apart each other. In a formal sense, each peak potential is equivalent to the standard redox potential of an MPC "redox couple" with adjacent valence states. Therefore, an MPC can be regarded as a multivalent redox species exhibiting plentiful standard redox potentials. For simplicity, we shall denote MPCs in the initial state as $\mathrm{MPC}^{Z}$, which can be reduced to $\mathrm{MPC}^{Z-1}$ and oxidized to $\mathrm{MPC}^{Z+1}$.

A clear feature of the responses in Figure 3 is the evolution of the photocurrent from negative to positive values with increasing Galvani potential difference. Previous works have shown that the excited porphyrin complex can be heterogeneously quenched by both electron acceptors (such as quinones) and electron donors (such as ferrocene derivatives). ${ }^{35,44}$ As MPCs can function as both electron acceptors and electron donors, the heterogeneous electron transfer across the liquid/liquid interface takes place in both directions:

$$
\begin{aligned}
& \mathrm{S}^{*}(\mathrm{w})+\mathrm{MPC}^{Z}(\mathrm{o}) \rightarrow \mathrm{S}^{+}(\mathrm{w})+\mathrm{MPC}^{Z-1}(\mathrm{o}) \\
& \mathrm{S}^{*}(\mathrm{w})+\operatorname{MPC}^{Z}(\mathrm{o}) \rightarrow \mathrm{S}^{-}(\mathrm{w})+\mathrm{MPC}^{Z+1}(\mathrm{o})
\end{aligned}
$$

where $\mathrm{S}^{*}$ corresponds to the photoexcited porphyrin complex in the aqueous phase, and $\mathrm{S}^{+}$and $\mathrm{S}^{-}$denote the oxidized and reduced states of the porphyrin complex. Under the potentiostatic conditions used in this work, the heterogeneous electron transfer reaction described by eq 1 results in a negative current when the interface is illuminated. Similarly to the case of quinone quenchers, ${ }^{44}$ this reaction is favored when a negative Galvani potential difference is applied between the aqueous and organic phases. This behavior is reflected in the increase of the photocurrent magnitude when more negative potentials are applied (Figure 3). On the other hand, in the case of eq 2 the electron transfer manifests itself as positive photocurrents, which are expected to increase when the potential is set to more positive values. ${ }^{35}$

When the Galvani potential difference is tuned from a negative value to more positive values, the negative response decreases while positive photocurrents become dominant. These two separate dependences of the kinetics of eqs 1 and 2 on the Galvani potential difference are in line with the published data on heterogeneous quenching of porphyrin complex by quinones ${ }^{44}$ or ferrocene derivatives. ${ }^{35}$ However, in the present case the two reactions occur simultaneously, which results in the convoluted responses observed at intermediate potentials. The magnitude and shape of the photocurrent transient are determined by the balance between the kinetics of these two

(43) Kamat, P. V. J. Phys. Chem. B 2002, 106, 7729-7744.

(44) Eugster, N.; Fermin, D. J.; Girault, H. H. J. Am. Chem. Soc. 2003, 125, $4862-4869$.

(45) Su, B.; Girault, H. H. J. Phys. Chem. B 2005, 109, 11427-11431. 


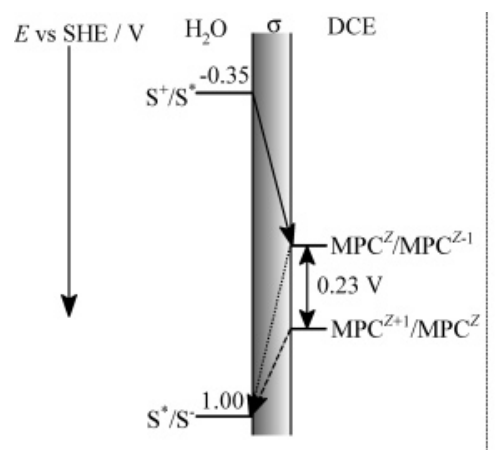

(a)

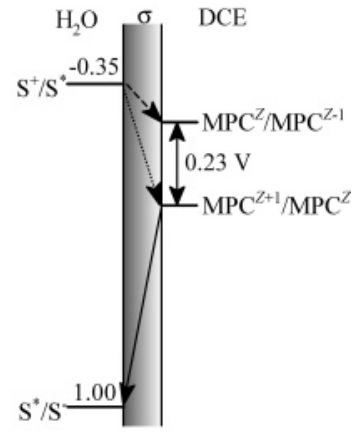

(b)
Figure 4. Schematic representation of the photoinduced electron-transfer reactions: (a) electron injection from the porphyrin complex to $\mathrm{MPC}^{Z}$; (b) electron injection from $\mathrm{MPC}^{Z}$ to the porphyrin complex. The interfacial region is denoted as $\sigma$. The solid, dotted, and dashed arrows denote the dominant electron-transfer reaction, the charge recombination reaction, and the opposite electron-transfer reaction, respectively.

reactions. This phenomenon can be rationalized by the scheme in Figure 4, which illustrates the electron-transfer process for $\mathrm{MPC}^{Z}$ reduction (a) as well as oxidation (b). The relative difference in redox potential between the porphyrin complex and $\mathrm{MPC}^{Z}$ depends on the applied potential. For instance, it can be considered that the situation in Figure $4 \mathrm{a}$ occurs when a negative Galvani potential difference is applied between the two phases, while Figure $4 \mathrm{~b}$ corresponds to the case of positive potentials. The solid arrows show the dominant electron transfer reaction in each case. From the diagrams in Figure 4, the formal Gibbs energies for reactions 1 and 2 can be defined, respectively, as

$$
\begin{gathered}
\left.\Delta G_{\mathrm{et}, 1}^{\circ \prime}=F\left(\left[E_{\left.\mathrm{S}^{+} / \mathrm{S}^{*}\right]_{\mathrm{SHE}}-\mathrm{w}}^{\mathrm{o}}\right]_{\mathrm{MPCZ} / \mathrm{MPCZ}-1}\right]_{\mathrm{SHE}}+\Delta_{\mathrm{o}}^{\mathrm{w}} \phi\right)= \\
F\left(\Delta_{\mathrm{o}}^{\mathrm{w}} \phi-\Delta_{\mathrm{o}}^{\mathrm{w}} \phi_{\mathrm{et}, 1}^{\circ \prime}\right) \\
\Delta G_{\mathrm{et}, 2}^{\circ \prime}=F\left(\left[E_{\mathrm{MPCZ}+1 / \mathrm{MPC}}^{\circ \prime, \mathrm{o}}\right]_{\mathrm{SHE}}-\left[E_{\mathrm{S}^{*} / \mathrm{S}^{-}}^{\circ, \mathrm{w}}\right]_{\mathrm{SHE}}-\Delta_{\mathrm{o}}^{\mathrm{w}} \phi\right)= \\
-F\left(\Delta_{\mathrm{o}}^{\mathrm{w}} \phi-\Delta_{\mathrm{o}}^{\mathrm{w}} \phi_{\mathrm{et}, 2}^{\circ \prime}\right)
\end{gathered}
$$

where $\left[E_{\mathrm{S}^{+} / \mathrm{S}^{*}}^{\mathrm{o}, \mathrm{w}}\right]_{\mathrm{SHE}},\left[E_{\mathrm{S}^{*} / \mathrm{S}^{-}}^{\circ, \mathrm{W}}\right]_{\mathrm{SHE}}$ are the formal redox potentials of the excited porphyrin complex in water and $\left[E_{\mathrm{MPCZ} / \mathrm{MPCZ}^{-1}}^{\mathrm{o}, \mathrm{o}}\right]_{\mathrm{SHE}},\left[E_{\mathrm{MPCZ}+1 / \mathrm{MPCZ}}^{\mathrm{o}, \mathrm{o}}\right]_{\mathrm{SHE}}$ those of MPCs in DCE. $\Delta_{\mathrm{o}}^{\mathrm{w}} \phi_{\mathrm{et}, 1}^{\circ \prime}$ and $\Delta_{\mathrm{o}}^{\mathrm{w}} \phi_{\mathrm{et}, 2}^{\circ \prime}$ correspond to the formal electron-transfer potentials. When the potential is varied from negative to positive values (i.e., from Figure 4a to Figure 4b), the redox potential of $\mathrm{MPC}^{Z}$ shifts to more negative values relative to that of the porphyrin complex in water. Consequently, the Gibbs energy associated with the oxidation of $\mathrm{MPC}^{Z}$ (reaction 2) gradually increases while that of eq 1 decreases. The dependence of the electron-transfer rate constants on the Gibbs energies expressed by eqs 3 and 4 gives rise to the changes in photocurrent responses observed in Figure 4.

The dashed arrows in Figure 4 illustrate the opposite processes, which feature slower kinetics due to the lower energy associated with these reactions. The dotted arrows represent charge recombination reactions. Directly after the heterogeneous electron-transfer event, the products are expected to remain in close contact as geminate ion pairs $\left\{\mathrm{S}^{+} \cdots \mathrm{MPC}^{\mathrm{Z}-1}\right\}$ or $\left\{\mathrm{S}^{-} \cdots \mathrm{MPC}^{\mathrm{Z}+1}\right\}$ at the interface. As described in the next section, these species are involved in two competing processes: back charge transfer leading to the starting species or separation to the products of reactions 1 and 2 . In the case of molecular quenchers, back charge transfer was observed to proceed as a first-order reaction with the time constant in the millisecond range. ${ }^{35}$

Kinetic Analysis of the Photocurrent Transient Responses. According to the scheme in Figure 4, the photoinduced electrontransfer process can be described by the following set of reactions:

Excitation and relaxation of the porphyrin complex:

$$
\mathrm{S} \underset{k_{\mathrm{d}}}{\stackrel{h v}{\rightleftarrows}} \mathrm{S}^{*}
$$

Heterogeneous electron-transfer reactions:

$$
\begin{aligned}
& \mathrm{S}^{*}+\mathrm{MPC}^{Z} \stackrel{k_{1}^{\mathrm{e}, \mathrm{II}}}{\longrightarrow}\left\{\mathrm{S}^{+} \cdots \mathrm{MPC}^{Z-1}\right\} \\
& \mathrm{S}^{*}+\mathrm{MPC}^{Z} \stackrel{k_{2}^{\mathrm{e}, \mathrm{II}}}{\longrightarrow}\left\{\mathrm{S}^{-} \cdots \mathrm{MPC}^{Z+1}\right\}
\end{aligned}
$$

Recombination reactions:

$$
\begin{aligned}
& \left\{\mathrm{S}^{+} \cdot \cdots \mathrm{MPC}^{Z-1}\right\} \stackrel{k_{1}^{\text {rec }}}{\longrightarrow} \mathrm{S}+\mathrm{MPC}^{Z} \\
& \left\{\mathrm{~S}^{-} \cdot \cdot \mathrm{MPC}^{Z+1}\right\} \stackrel{k_{2}^{\text {rec }}}{\longrightarrow} \mathrm{S}+\mathrm{MPC}^{Z}
\end{aligned}
$$

Separation of the products:

$$
\begin{aligned}
& \left\{\mathrm{S}^{+} \cdot \cdots \mathrm{MPC}^{\mathrm{Z}-1}\right\} \stackrel{k \mathrm{ps}}{\longrightarrow} \mathrm{S}^{+}+\mathrm{MPC}^{Z-1} \\
& \left\{\mathrm{~S}^{-} \cdot \cdots \mathrm{MPC}^{Z+1}\right\} \stackrel{k \mathrm{~kg}^{\mathrm{s}}}{\longrightarrow} \mathrm{S}^{-}+\mathrm{MPC}^{Z+1}
\end{aligned}
$$

Back electron-transfer reactions:

$$
\begin{aligned}
& \mathrm{S}^{+}+\mathrm{MPC}^{Z} \stackrel{k_{1}^{\mathrm{b}, I}}{\longrightarrow} \mathrm{S}+\mathrm{MPC}^{Z+1} \\
& \mathrm{~S}^{-}+\mathrm{MPC}^{Z} \stackrel{k_{2}^{\mathrm{k}, I}}{\longrightarrow} \mathrm{S}+\mathrm{MPC}^{Z-1}
\end{aligned}
$$

On the basis of our previous formulation of photocurrent responses ${ }^{46}$ and considering the relatively low magnitude of the signals in Figure 3, the concentrations of porphyrin complex and $\mathrm{MPC}^{Z}$ are taken to be constant. This allows describing the various charge-transfer processes as pseudo-first-order reactions with rate constants expressed by

$$
\begin{aligned}
k^{\mathrm{et}} & =k^{\mathrm{et}, \mathrm{II}}\left[\mathrm{MPC}^{\mathrm{Z}}\right] \\
k^{\mathrm{b}} & =k^{\mathrm{b}, \mathrm{II}}\left[\mathrm{MPC}^{Z}\right]
\end{aligned}
$$

It should be noted that, in the case of molecular quenchers such as quinones or ferrocene, reactions 12 and 13 do not exist. The terms recombination and back electron transfer were used indiscriminately to refer to reactions 8 and 9. In the present case, however, these two terms correspond to different mechanisms. Taking the contributions from all the elementary steps, i.e., reactions 5-13, the photocurrent density as a function of time is obtained (Supporting Information):

(46) Samec, Z.; Eugster, N.; Fermin, D. J.; Girault, H. H. J. Electroanal. Chem. 2005, 577, 323-337. 


$$
\begin{aligned}
j= & g_{1} F \cdot \\
& \left\{\frac{k_{1}^{\mathrm{ps}} \exp \left(-k_{1}^{\mathrm{b}} t\right)+\left(k_{1}^{\mathrm{rec}}-k_{1}^{\mathrm{b}}\right) \exp \left[-\left(k_{1}^{\mathrm{rec}}+k_{1}^{\mathrm{ps}}\right) t\right]}{\left(k_{1}^{\mathrm{rec}}+k_{1}^{\mathrm{ps}}-k_{1}^{\mathrm{b}}\right)}\right\}+ \\
& +g_{2} F \cdot\left\{\frac{k_{2}^{\mathrm{ps}} \exp \left(-k_{2}^{\mathrm{b}} t\right)+\left(k_{2}^{\mathrm{rec}}-k_{2}^{\mathrm{b}}\right) \exp \left[-\left(k_{2}^{\mathrm{rec}}+k_{2}^{\mathrm{ps}}\right) t\right]}{\left(k_{2}^{\mathrm{rec}}+k_{2}^{\mathrm{ps}}-k_{2}^{\mathrm{b}}\right)}\right\}
\end{aligned}
$$

The parameters $g_{1}$ and $g_{2}$ correspond to the fluxes of electron injection associated with the electron-transfer reactions 6 and 7:

$$
\begin{aligned}
& g_{1}=I_{0} \sigma \Gamma_{\mathrm{S}} \frac{-k_{1}^{\mathrm{et}}}{k_{\mathrm{d}}+k_{1}^{\mathrm{et}}+k_{2}^{\mathrm{et}}} \\
& g_{2}=I_{0} \sigma \Gamma_{\mathrm{S}} \frac{k_{2}^{\mathrm{et}}}{k_{\mathrm{d}}+k_{1}^{\mathrm{et}}+k_{2}^{\mathrm{et}}}
\end{aligned}
$$

where $I_{0}$ is the incident photo flux, $\sigma$, the optical capture cross section, and $\Gamma_{\mathrm{S}}$, the surface excess of porphyrin complex. The parameter $\Gamma_{\mathrm{S}}$ and $\sigma$ can be taken as $4.0 \times 10^{-10} \mathrm{~mol} \mathrm{~cm}^{-2}$ and $1.0 \times 10^{-17} \mathrm{~cm}^{2}$, respectively. ${ }^{35}$ Previous studies have shown that $\Gamma_{\mathrm{S}}$ is effectively potential independent over the range of Galvani potential differences used in Figure 3. ${ }^{35,47}$ The incident photon flux, $I_{0}$, is $6.4 \times 10^{15} \mathrm{~cm}^{-2} \mathrm{~s}^{-1}$. Based on time-resolved EPR spectroscopy and flash photolysis measurements at the water/DCE interface in total internal reflection, $k_{\mathrm{d}}$ is of the order of $5.0 \times 10^{5} \mathrm{~s}^{-1}$. Knowing this, it is possible to extract information on the dependence of $k_{1}^{\text {et }}$ and $k_{2}^{\text {et }}$ on $\Delta_{\mathrm{o}}^{\mathrm{w}} \phi$ from the values of the initial photocurrent $j_{0}$. This analysis does not take into account attenuation effects resulting from the uncompensated resistance, which will significantly complicate eq 16 . As the photocurrent responses in Figure 3 appear very sharp, only minimal errors are introduced by this simplification. Considering that a purely negative response is obtained at $-0.15 \mathrm{~V}$, a simulation using our previously published model shows an error of less than $5 \%$ on the value of $j_{0}{ }^{46}$ Finally, it should be mentioned that the formation of other oxidation states of MPCs, such as the photo-oxidation of $\mathrm{MPC}^{\mathrm{Z}+1}$ to $\mathrm{MPC}^{\mathrm{Z}+2}$, was not considered either. Since the successive redox potentials of the MPCs are separated by more than $200 \mathrm{mV}$, the oxidation of $\mathrm{MPC}^{Z+1}$ to $\mathrm{MPC}^{\mathrm{Z}+2}$ is expected to be slower than that of $\mathrm{MPC}^{\mathrm{Z}}$ to $\mathrm{MPC}^{Z+1}$. Furthermore, the concentration of $\mathrm{MPC}^{Z+1}$ remains much lower than that of $\mathrm{MPC}^{Z}$ at realistic illumination levels. Hence, these additional reaction pathways are expected to generate very little current, which is neglected for simplicity.

Figure 5 illustrates the deconvolution of the photocurrent transient obtained at $-0.05 \mathrm{~V}$. The dotted line is the experimental curve. The dashed lines represent the negative and positive contributions to the photocurrent, which are calculated from the first and second terms of eq 16, respectively. The solid line represents the summation of the two dashed lines, corresponding to the net simulated photocurrent response. It can be clearly seen that the experimental curve amounts to the sum of two photocurrent transients related to the electron transfers in two directions. The initial value of the photocurrent, observed

(47) Eugster, N. Ph.D. Thesis, Ecole Polytechnique Fédérale de Lausanne, 2004.

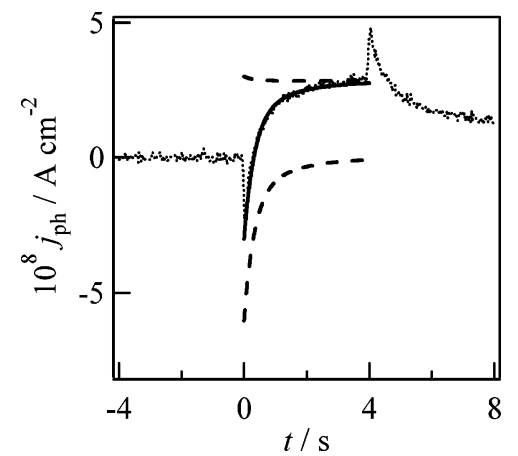

Figure 5. Simulation of the photocurrent transient at $0.05 \mathrm{~V}$. The dotted line corresponds to the experimental trace. The solid line was calculated from eq 16 using the following parameters: $\Gamma_{\mathrm{S}}=4.0 \times 10^{-10} \mathrm{~mol} \mathrm{~cm}^{-2}$, $\sigma=1.0 \times 10^{-17} \mathrm{~cm}^{2}, I_{0}=6.4 \times 10^{15} \mathrm{~cm}^{-2} \mathrm{~s}^{-1}, k_{\mathrm{d}}=5.0 \times 10^{5} \mathrm{~s}^{-1}, k_{1}^{\text {et }}=$ $9.2 \times 10^{3} \mathrm{~s}^{-1}, k_{2}^{\mathrm{et}}=4.6 \times 10^{3} \mathrm{~s}^{-1}, k_{1}^{\mathrm{ps}}=0.5 \mathrm{~s}^{-1}, k_{2}^{\mathrm{ps}}=3.5 \mathrm{~s}^{-1}, k_{1}^{\mathrm{rec}}=2.5$ $\mathrm{s}^{-1}, k_{2}^{\text {rec }}=0.2 \mathrm{~s}^{-1}, k_{1}^{\mathrm{b}}=0.7 \mathrm{~s}^{-1}$, and $k_{2}^{\mathrm{b}}=0.01 \mathrm{~s}^{-1}$. The two dashed lines illustrate the positive and negative contributions to the net photocurrent calculated from the second and first term of eq 16, respectively.

directly at the beginning of the illumination, is determined by the sum of the fluxes of electron injection multiplied by Faraday's constant:

$$
j_{0}=F\left(g_{1}+g_{2}\right)=I_{0} \sigma \Gamma_{\mathrm{S}} F \frac{k_{2}^{\mathrm{et}}-k_{1}^{\mathrm{et}}}{k_{\mathrm{d}}+k_{1}^{\mathrm{et}}+k_{2}^{\mathrm{et}}}
$$

The pseudo-first-order electron-transfer rate constants $k_{1}^{\text {et }}=$ $9.2 \times 10^{3} \mathrm{~s}^{-1}$ and $k_{2}^{\mathrm{et}}=4.6 \times 10^{3} \mathrm{~s}^{-1}$ were used to simulate the experimental transient in Figure 5. These values confirm that the two processes occur simultaneously with comparable rates. These rate constants remain 1 or 2 orders of magnitude lower than the rate of relaxation of the excited state of the porphyrin complex. Clearly, the large number of independent variables contained in eq 16 prevents an accurate fitting of the photocurrent transients. The purpose of Figure 5 is to illustrate the decomposition of the photocurrent response into two separate contributions. We shall not present an analysis of the dependence of the recombination and back electron transfer rates. Rather, we shall concentrate on the initial photocurrent values to extract information on the kinetics of the photoinduced electron-transfer process.

The decay features observed on both the positive and negative contributions to the photocurrent in Figure 5 are determined by the rate constants $k^{\mathrm{ps}}, k^{\mathrm{b}}$, and $k^{\mathrm{rec}}$. The back electron transfer and charge recombination mechanisms also give rise to the positive overshoot observed in Figure 5 after the illumination is stopped. The presence of these two reactions contributing to the photocurrent relaxation gives rise to multiexponential decays for both the positive and negative contributions.

The dependence of the rate constant of heterogeneous electron transfer on the formal Gibbs energy in the case of molecular quenchers was found to follow Marcus theory. However, in the present case, the relatively narrow range of $\Delta G_{\mathrm{et}, 1}^{\circ}$ and $\Delta G_{\mathrm{et}, 2}^{\circ \prime}$ values prevents a similar analysis. Rather, we shall consider that a fraction $\alpha$ of the Galvani potential difference acts to lower the activation barrier for the electron-transfer processes. As a first approximation, the same apparent charge-transfer coefficient can be taken for reactions 6 and 7, since both proceed from the same reactants. Within the framework of Butler-Volmer 

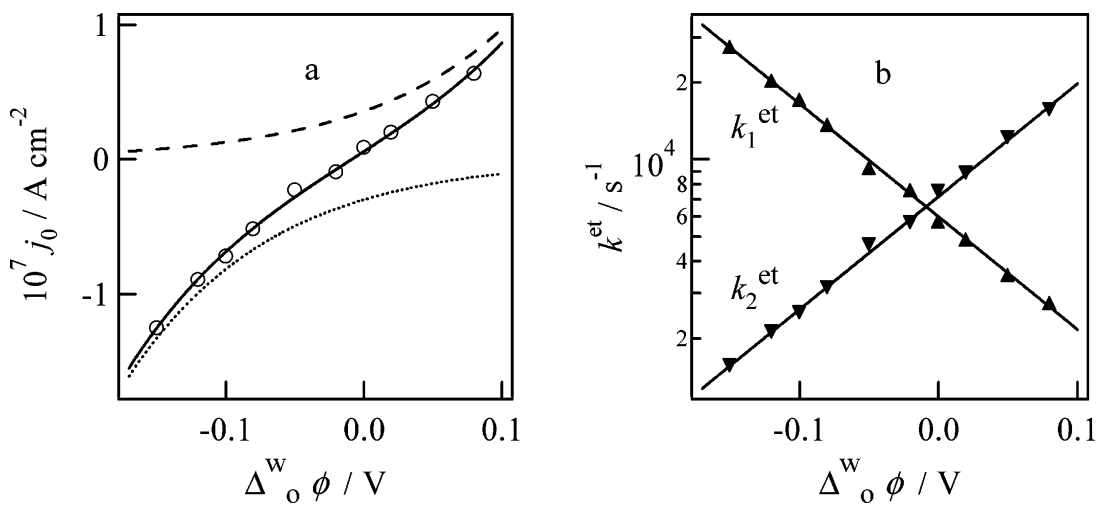

Figure 6. (a) Initial photocurrent $j_{0}$ as a function of the Galvani potential difference, as estimated from the data in Figure 3 . The solid line is a fit to eq 19 with the rate constants calculated by eqs 22 and 23 . The fitting parameters were $k_{1}^{\mathrm{et}, \mathrm{o}}=6.0 \times 10^{3} \mathrm{~s}^{-1}, k_{2}^{\mathrm{et}, \mathrm{o}^{\prime}}=7.2 \times 10^{3} \mathrm{~s}^{-1}$ and $\alpha=0.26$. The dotted and dashed lines illustrate the positive and negative contributions to $j_{0}$, calculated as $F g_{2}$ and $F g_{1}$, respectively. (b) Apparent rate constants of the heterogeneous electron-transfer reactions as a function of the Galvani potential difference. The solid lines are calculated from eqs 22 and 23 with the parameters obtained in (a).

approximation, the pseudo-first-order rate constants for reactions 6 and 7 are given by

$$
\begin{gathered}
k_{1}^{\mathrm{et}}=k_{1}^{\mathrm{et}, \mathrm{o}} \exp \left(-\frac{\alpha F\left(\Delta_{\mathrm{o}}^{\mathrm{w}} \phi-\Delta_{\mathrm{o}}^{\mathrm{w}} \phi_{\mathrm{et}, 1}^{\circ}\right)}{R T}\right)=k_{1}^{\mathrm{et}, \mathrm{o} \prime} \exp \left(-\frac{\alpha F \Delta_{\mathrm{o}}^{\mathrm{w}} \phi}{R T}\right) \\
k_{2}^{\mathrm{et}}=k_{2}^{\mathrm{et}, \mathrm{o}} \exp \left(\frac{\alpha F\left(\Delta_{\mathrm{o}}^{\mathrm{w}} \phi-\Delta_{\mathrm{o}}^{\mathrm{w}} \phi_{\mathrm{et}, 2}^{\circ \prime}\right)}{R T}\right)=k_{2}^{\mathrm{et}, \mathrm{o} \prime} \exp \left(\frac{\alpha F \Delta_{\mathrm{o}}^{\mathrm{w}} \phi}{R T}\right)
\end{gathered}
$$

where $k_{1}^{\text {et,o }}$ and $k_{2}^{\text {et,o }}$ are the standard rate constants of reactions 6 and 7, respectively. It is more convenient, however, to refer to the apparent rate constants $k_{1}^{\text {et,o' }}$ and $k_{2}^{\text {et,o' }}$ at zero Galvani potential difference $\left(\Delta_{\mathrm{o}}^{\mathrm{w}} \phi=0\right)$. Then the following Tafel relations can be obtained:

$$
\begin{aligned}
& \ln k_{1}^{\mathrm{et}}=\ln k_{1}^{\mathrm{et}, \mathrm{o} \prime}-\frac{\alpha F \Delta_{\mathrm{o}}^{\mathrm{w}} \phi}{R T} \\
& \ln k_{2}^{\mathrm{et}}=\ln k_{2}^{\mathrm{et}, \mathrm{o} \prime}+\frac{\alpha F \Delta_{\mathrm{o}}^{\mathrm{w}} \phi}{R T}
\end{aligned}
$$

Employing eqs 19, 22, and 23, the initial photocurrent $j_{0}$ can be expressed as a function of the Galvani potential difference, the apparent charge-transfer coefficient $\alpha$, and the apparent rate constants $k_{1}^{\mathrm{et}, \mathrm{o} \prime}$ and $k_{2}^{\mathrm{et}, \mathrm{o} \prime}$. The solid line in Figure $6 \mathrm{a}$ is a fit of the experimental initial photocurrent as a function of the Galvani potential difference employing this system of equations. This analysis yields apparent rate constants at $\Delta_{\mathrm{o}}^{\mathrm{w}} \phi=0$ of $k_{1}^{\mathrm{et}, \mathrm{o} \prime}=$ $6.0 \times 10^{3} \mathrm{~s}^{-1}, k_{2}^{\mathrm{et}, \mathrm{o}^{\prime}}=7.2 \times 10^{3} \mathrm{~s}^{-1}$, and an apparent chargetransfer coefficient of $\alpha=0.26$. It can be observed that $k_{\mathrm{d}} \gg$ $k_{1}^{\text {et }}, k_{2}^{\text {et }}$ throughout the potential range. Therefore, the initial photocurrent as described by eq 19 appears proportional to $k_{2}^{\text {et }}$ $-k_{1}^{\text {et }}$ with a proportionality constant of $I_{0} \sigma \Gamma_{\mathrm{S}} F / k_{\mathrm{d}}$, and errors in the estimation of these parameters should not affect the trend observed in Figure 6a.

The radius of the porphyrin complex is $0.7 \mathrm{~nm},{ }^{35}$ and that of $\mathrm{MPC}^{Z}$ can be taken as a sum of the metallic core $(0.8 \mathrm{~nm})$ and the protecting monolayer thickness of $0.8 \mathrm{~nm},{ }^{7}$ which yields a value of $1.6 \mathrm{~nm}$. Therefore, it is reasonable to consider a distance of at least $2.3 \mathrm{~nm}$ separating the porphyrin complex from the $M C^{Z}$. This distance is significantly larger than the thickness of the interfacial layer, which was estimated to be $1 \mathrm{~nm}$ by molecular dynamic simulation ${ }^{48,49}$ and spectroscopic techniques. ${ }^{50,51}$ However, the clear dependence of $k_{\text {et }}$ on the Galvani potential difference indicates that a substantial portion of the Galvani potential difference is developed within the average distance between the porphyrin complex and $\mathrm{MPC}^{Z}$. The obtained value of $\alpha=0.26$ suggests that the potential profile across the interface extends over a region much wider than the solvent profile, which has been predicted previously by the simulations of the ionic distribution based on the GouyChapman ${ }^{52}$ and lattice-gas models. ${ }^{53-55}$ Further studies will be aimed at controlling the distance separating the redox species by variation of the chain length of the protecting alkanethiolate. By exploring the effect of this parameter on the heterogeneous electron-transfer rate constant, we expect to obtain further insight into the potential distribution at liquid/liquid interfaces.

Figure $6 \mathrm{~b}$ shows the rate constants of heterogeneous electron transfer $k_{1}^{\text {et }}$ and $k_{2}^{\text {et }}$ as extracted from the data in Figure 6a. At $-0.15 \mathrm{~V}, k_{1}^{\text {et }}$ was estimated to be ca. $2.7 \times 10^{4} \mathrm{~s}^{-1}$, which corresponds to a heterogeneous pseudo-first-order rate constant of $6.8 \times 10^{-3} \mathrm{~cm} \mathrm{~s}^{-1}$ assuming a characteristic distance of 2.5 $\mathrm{nm}$ separating the redox species at the interface. The corresponding value of the apparent bimolecular rate constant is estimated to be $k_{1}^{\text {et,II }}=340 \mathrm{M}^{-1} \mathrm{~cm} \mathrm{~s}^{-1}$. This value is significantly larger than those reported for electron-transfer reactions between conventional redox molecules at liquid/liquid interfaces, ${ }^{56-58}$ indicating a fast heterogeneous electron-transfer reaction between the porphyrin complex and the MPC. Our present results contradict those reported by Quinn et al. in a recent study, ${ }^{11}$ in which the rates of the heterogeneous electron transfer reactions between MPCs and various aqueous redox species were reported to be too slow to measure by scanning electrochemical microscopy (SECM) under feedback operation

(48) Benjamin, I. Chem. Rev. 1996, 96, 1449-1475

(49) Benjamin, I. Annu. Rev. Phys. Chem. 1997, 48, 407-451.

(50) Strutwolf, J.; Zhang, J.; Barker, A. L.; Unwin, P. R. Phys. Chem. Chem Phys. 2001, 3, 5553-5558.

(51) Webster, R. D.; Beaglehole, D. Phys. Chem. Chem. Phys. 2000, 2, 56605666.

(52) Samec, Z. Chem. Rev. 1988, 88, 617-632.

(53) Frank, S.; Schmickler, W. J. Electroanal. Chem. 2000, 483, 18-21.

(54) Frank, S.; Schmickler, W. J. Electroanal. Chem. 2001, 500, 491-497.

(55) Schmickler, W. J. Electroanal. Chem. 1997, 428, 123-127.

(56) Chen, S.; Huang, K. J. Cluster Sci. 2000, 11, 405-422.

(57) Tsionsky, M.; Bard, A. J.; Mirkin, M. V. J. Am. Chem. Soc. 1997, 119, 10785-10792.

(58) Zhang, J.; Barker, A. L.; Unwin, P. R. J. Electroanal. Chem. 2000, 483, 95-107. 
mode even with the driving force extending over $1.3 \mathrm{~V}$. It should be stressed that the MPCs used in their study are identical to those used in the present work, both containing a sufficiently dominant population of $\mathrm{Au}_{140}$ cores $^{10}$ with a diameter of $\sim 1.6$ $\mathrm{nm}$. On the other hand, the rate constants obtained here are comparable with that of $76 \mathrm{M}^{-1} \mathrm{~cm} \mathrm{~s}^{-1}$ reported by Georganopoulou et al. ${ }^{29}$ for the case of the heterogeneous electrontransfer reaction between $\mathrm{Au}_{38} \mathrm{MPC}$ and $\mathrm{IrCl}_{6}{ }^{2-}$ at the water/ DCE interface employing SECM technique. Indeed, considering an MPC concentration of $0.02 \mathrm{mM}$ and a distance of $2.5 \mathrm{~nm}$ between the reactant centers, the bimolecular electron-transfer rate constants at $\Delta_{\mathrm{o}}^{\mathrm{w}} \phi=0$ can be calculated as $75 \mathrm{M}^{-1} \mathrm{~cm} \mathrm{~s}^{-1}$ and $90 \mathrm{M}^{-1} \mathrm{~cm} \mathrm{~s}^{-1}$ for the reduction and oxidation, respectively. However, it should be noted that the electronic properties of $\mathrm{Au}_{140}$ and $\mathrm{Au}_{38} \mathrm{MPCs}$ are different. It has been shown that $\mathrm{Au}_{38}$ is small enough $(d \sim 1.1 \mathrm{~nm})$ to exhibit a HOMO-LUMO energy gap, representing molecule-like redox activity.,40,59-61 Therefore, in the case of $\mathrm{Au}_{38}$, evaluation of the electron transfer rate constant can be carried out using the approach previously developed for electron-transfer reactions between traditional redox molecules. Due to the larger size of $\mathrm{Au}_{140}$ compared with conventional molecules, as well as the possibility of successive electron transfer, bimolecular rate constants obtained with these species should be considered with caution.

(59) Lee, D.; Donkers, R. L.; DeSimone, J. M.; Murray, R. W. J. Am. Chem. Soc. 2003, 125, 1182-1183.

(60) Jimenez, V. L.; Georganopoulou, D. G.; White, R. J.; Harper, A. S.; Mills, A. J.; Lee, D.; Murray, R. W. Langmuir 2004, 20, 6864-6870.

(61) Yang, Y.; Chen, S. Nano Lett. 2003, 3, 75-79.

\section{Conclusions}

MPCs were used as redox mediators in heterogeneous photocurrent generation at the polarized water/DCE interface. Photocurrent responses originating from the heterogeneous electron transfer between photoexcited porphyrin complexes and MPCs were studied. As MPCs can function as both electron acceptors and electron donors, heterogeneous electron transfer can take place in either direction. The balance between reduction and oxidation of MPC can be controlled by tuning the Galvani potential difference across the interface. At intermediate potentials, photoinduced electron transfer in the two directions occurs simultaneously with comparable rates. Analysis of the initial photocurrent values allows addressing the dependence of the rate constants of heterogeneous electron transfer on the Galvani potential difference. The effect of the Galvani potential difference on the photocurrent responses is adequately described by a Butler-Volmer type dependence.

Acknowledgment. The work was supported by the grants from the Fonds National Suisse de la Recherche Scientifique and EPFL. The technical assistance by Valérie Devaud is acknowledged.

Supporting Information Available: Mathematical development of photocurrent density as a function of time (eq 16). This material is available free of charge via the Internet at http://pubs.acs.org.

JA052415W 